\title{
Composição química, valores de energia metabolizável e aminoácidos digestíveis de subprodutos do arroz para frangos de corte
}

\author{
Chemical composition, metabolizable energy and digestible amino acids values of rice by-products \\ for broilers
}

\author{
Otto Mack Junqueira ${ }^{\mathrm{I}^{*}}$ Karina Ferreira Duarte ${ }^{\mathrm{I}}$ Luciana Cardoso Cancherini ${ }^{\mathrm{II}}$ \\ Lúcio Francelino Araújo ${ }^{\mathrm{III}}$ Maria Cristina de Oliveira ${ }^{\mathrm{IV}}$ Edivaldo Antônio Garciav $^{\mathrm{V}}$
}

RESUMO

Dois experimentos foram conduzidos para determinar a composição química, os valores de energia e os coeficientes de digestibilidade dos aminoácidos, do farelo de arroz integral (FAI) e da quirera de arroz (QA). No primeiro estudo, foram utilizadas 144 aves, com 21 dias de idade, machos, linhagem Cobb, que tiveram suas excretas totalmente coletadas para determinação da energia metabolizável aparente (EMA) e energia metabolizável aparente corrigida (EMAn). O delineamento experimental foi inteiramente casualizado, com três tratamentos e seis repetições, com oito aves cada. No segundo experimento, foi utilizado o método de alimentação forçada para a determinação dos coeficientes de digestibilidade dos aminoácidos. O delineamento foi inteiramente casualizado, com dois alimentos e um jejum e seis repetições com um galo cada. Os valores de $M S, P B, E E$, $F B$, EMA e EMAn foram, respectivamente, para FAI: 88,6\%; $11,8 \%$; 15,3\%; 10,2\%; 2968kcal kg-1 e 2804kcal kg-1 e para QA: 93,5\%; 9,1\%; 0,73\%; 0,45\%; 3338kcal kg-1 e 3239kcal $\mathrm{kg}^{-1}$. Os valores médios encontrados dos coeficientes de digestibilidade de aminoácidos essenciais e não essenciais foram, respectivamente, de 75,9\% e 73,9\%, para FAI, e 77,9\% e 76,5\%, para QA. Embora tenham apresentado níveis inferiores de energia, FAI e a QA podem ser utilizados nas rações de aves em substituição ao milho, uma vez que tiveram níveis maiores de proteína bruta e aminoácidos digestíveis.

Palavras-chave: coeficientes de digestibilidade, farelo de arroz, galos cecectomizados, quirera de arroz.

\begin{abstract}
Two experiments were conducted to determine the chemical composition, energy and the digestibility coefficients of the amino acids of the whole rice meal (WRM) and broken rice $(B R)$. In the first trial, one hundred forty four broilers, 21 days of age, male, of Cobb strain had their excreta totally collected in order to determine the apparent metabolizable energy (AME) and corrected apparent metabolizable energy (AMEn). The experimental design was completely randomized, with three treatments and six replicates with eight birds each one. In the second trial, it was used the forced feeding method with cecectomized cockerels to determine the digestibility coefficients of the amino acids. The design was entirely randomized, with two feeds and one fasting and six replicates with one bird each one. The values of DM, CP, EE, CF, AME and AMEn were, respectively, to WRM: $88.6 \% ; 11.8 \%$; $15.3 \%$;

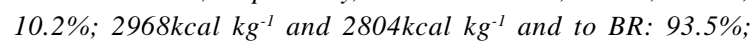
9.1\%; $0.73 \% ; 0.45 \%$; 3338kcal $\mathrm{kg}^{-1}$ and $3239 \mathrm{kcal}^{-1}$. The average values of digestibility coefficients of essential and nonessential amino acids were, respectively, $75.9 \%$ and $73.9 \%$ to WRM and $77.9 \%$ and $76.5 \%$ to BR. The WRM and the BR can be used in the diets of broilers in substitution to the corn, however showed lower metabolizable energy levels, had higher levels of crude protein and digestible amino acid.
\end{abstract}

Key words: broken rice, cecectomized cockerels, digestibility coefficients, whole rice meal.

IDepartamento de Zootecnia, Faculdade de Ciências Agrárias e Veterinárias (FCAV), Universidade Estadual Paulista (UNESP). Via de Acesso Prof. Paulo Donato Castellane, s/n, 14884-900, Jaboticabal, SP, Brasil. E-mail: ottomack@fcav.unesp.br. *Autor para correspondência.

IIDepartamento de Zootecnia, Universidade do Estado de Mato Grosso (UNEMAT), Campus Universitário de Pontes e Lacerda, Pontes e Lacerda, MT, Brasil.

IIIDepartamento de Zootecnia, Universidade de São Paulo (USP), Faculdade de Zootecnia e Engenharia de Alimentos (FZEA), Pirassununga, SP, Brasil.

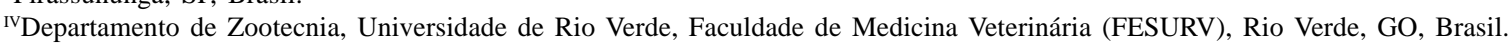

v'Departamento de Produção e Exploração Animal, Faculdade de Medicina Veterinária e Zootecnia (FMVZ), UNESP, Botucatu, SP, Brasil. 


\section{INTRODUÇÃO}

À medida que os custos de produção aumentam, os nutricionistas têm a necessidade de buscar novas alternativas que possam reduzir custos e atender às exigências dos animais nas suas diferentes fases de produção. A utilização de alimentos alternativos e subprodutos da indústria pode ser interessante sob o ponto de vista econômico na produção animal. Entretanto, para a formulação de rações nutricionalmente balanceadas, é de fundamental importância conhecer o valor nutritivo desses alimentos. Para tanto, é imprescindível o conhecimento da composição química e dos valores de energia, bem como a disponibilidade dos nutrientes. Dietas balanceadas de forma inadequada aumentam o consumo de ração, reduzem o ganho de peso e pioram a conversão alimentar, determinando aumento dos custos de produção (BRUM et al., 1999).

A contínua avaliação dos ingredientes é importante para manter atualizado um banco de dados, possibilitando melhorar as estimativas das médias de energia metabolizável e nutrientes que são utilizados nas dietas de aves. Essas variações que ocorrem na composição e no valor energético dos ingredientes são mais evidentes nos subprodutos, uma vez que a obtenção destes nem sempre é padronizada (BRUM et al., 2000).

A determinação das composições química e energética dos ingredientes torna-se muito importante, uma vez que vários fatores podem interferir no seu conteúdo de nutrientes e de energia. Dentre os fatores que determinam a variabilidade dos nutrientes e da energia dos grãos cereais, pode-se citar a fertilidade e o tipo de solo, o clima, a variedade da planta, o armazenamento, a amostragem, os tipos de processamento e os princípios antinutricionais (ALBINO, 1991).

Os aminoácidos que compõem a proteína dos ingredientes não são totalmente disponíveis para a absorção. Em função disso, vários estudos foram realizados com o intuito de verificar a digestibilidade de aminoácidos nos ingredientes (CAFÉ et al., 2000; VIEITES et al., 2000; RODRIGUES et al., 2002). O grande erro na elaboração de rações com base em aminoácidos totais é considerar que os alimentos possuem os mesmos coeficientes de digestibilidade dos aminoácidos, o que não é verdade (PARSONS, 1996).

O farelo de arroz apresenta características importantes, principalmente na sua composição química, como o alto teor de óleo, que possibilita sua utilização como fonte de energia para as aves em substituição ao milho (CONTE, 2000). ALI et al. (1998), ao analisarem o farelo de arroz de diferentes variedades, encontraram teores de óleo variando de 16,7 a 21,4\%. Já a quirera de arroz apresenta composição química semelhante ao milho, porém com menor teor de extrato etéreo.

O farelo de arroz integral pode conter variações nos teores de proteína, gordura, amido, dentre outros nutrientes. Assim, os valores de energia metabolizável podem apresentar muitas variações, tais como: 2.090kcal kg-1 (ROSTAGNO et al., 1994), 2.980kcal $\mathrm{kg}^{-1}$ (NRC, 1994) e $2.860 \mathrm{kcal} \mathrm{kg}^{-1}$ (ALBINO, 1991). A energia metabolizável aparente, determinada pela diferença entre a energia contida no alimento e a energia da excreta, apresenta, segundo RAMALHO (1998), alguns inconvenientes, dentre eles, a variação do valor energético em virtude do maior ou menor consumo do alimento e do alto custo da determinação e, principalmente, a consideração de que toda a energia excretada é proveniente do alimento, o que não é correto, pois parte é originada de produtos metabólicos e endógenos. Entretanto, a energia metabolizável aparente ainda é a mais utilizada nos cálculos de balanceamento de ração, em razão, principalmente, da falta de informações sobre os valores de energia metabolizável verdadeira, de acordo com ALBINO (1991).

Segundo ROSTAGNO et al. (2005), a quirera de arroz é um produto de alta qualidade que possui níveis protéicos e de energia metabolizável semelhantes aos do milho. Embora apresente um nível de gordura inferior ao do milho, ela compensa essa carência com o elevado teor de amido, além de apresentar um nível de fibra bruta inferior e lisina e metionina superiores, permitindo a formulação de dietas com menor nível de aminoácidos sintéticos, reduzindo assim o custo da ração.

O que dificulta o uso de alimentos não convencionais para aves, como a quirera e o farelo de arroz, é a escassez de trabalhos com determinação dos seus aminoácidos digestíveis. Considerando a importância dessas informações, este trabalho foi desenvolvido com o objetivo de determinar a composição química, a energia metabolizável e os aminoácidos digestíveis do farelo de arroz integral e da quirera de arroz para aves.

\section{MATERIAL E MÉTODOS}

Dois ensaios de metabolismo foram conduzidos com frangos de corte, com o objetivo de determinar a energia metabolizável aparente (EMA) e a aparente corrigida (EMAn) e os coeficientes de digestibilidade dos aminoácidos contidos no farelo de arroz integral (FAI) e na quirera de arroz (QA). 
Para a composição química dos ingredientes, foram determinados os valores de proteína bruta (PB), matéria seca (MS), extrato etéreo (EE), fibra bruta (FB), matéria mineral (MM), cálcio (Ca), fósforo total (Ptotal), sódio $(\mathrm{Na})$, lisina, metionina, ácido aspártico, treonina, triptofano, serina, glicina, alanina, valina, isoleucina, leucina, fenilalanina, histidina, arginina, cistina, ácido glutâmico e prolina.

Ensaio 1 - Determinação da energia metabolizável aparente (EMA) e aparente corrigida (EMAn)

Para a determinação da energia metabolizável, foi utilizada a metodologia de coleta total de excretas, utilizando-se 144 frangos de corte de 21 dias de idade, machos, da linhagem Cobb, distribuídos em um delineamento inteiramente casualizado, com três tratamentos e seis repetições, totalizando 18 parcelas experimentais compostas por oito aves.

As aves foram alojadas em gaiolas de metabolismo e receberam água e ração à vontade durante todo período experimental. Os tratamentos consistiram em uma dieta referência, formulada à base de milho e farelo de soja (Tabela 1), e o farelo de arroz integral (FAI) e a quirera (QA) substituíram, com base na matéria natural, 40\% da ração referência.

Tabela 1 - Composição percentual e níveis nutricionais da ração de referência.

\begin{tabular}{|c|c|}
\hline Ingredientes & $\%$ \\
\hline Milho grão & 62,81 \\
\hline Farelo de soja & 29,90 \\
\hline Fosfato bicálcico & 1,62 \\
\hline Calcário calcítico & 1,03 \\
\hline Óleo de soja & 3,47 \\
\hline Sal comum & 0,41 \\
\hline L-lisina $\mathrm{HCl}(78,8 \%)$ & 0,16 \\
\hline Suplemento mineral + vitamínico ${ }^{1}$ & 0,60 \\
\hline Total & 100,00 \\
\hline \multicolumn{2}{|c|}{------------------------Valores Calculados²- } \\
\hline Energia metabolizável (kcal kg ${ }^{-1}$ ) & 3.100 \\
\hline Proteína bruta (\%) & 19,000 \\
\hline Cálcio (\%) & 0,911 \\
\hline Fósforo disponível (\%) & 0,406 \\
\hline Sódio (\%) & 0,202 \\
\hline Lisina (\%) & 1,045 \\
\hline Metionina (\%) & 0,417 \\
\hline
\end{tabular}

1. Enriquecido por kg de ração: Vitaminas: (A 8000UI; $D_{3}$ 1800UI; E 12mg; K 2mg; B 1 1mg; $B_{2} 4 \mathrm{mg} ; B_{6} 1 \mathrm{mg} ; B_{12} 10 \mathrm{mcg}$ ); Piridoxina 400mg; Ácido Fólico 0,40mg; Biotina 0,04mg; Niacina 28mg; Ácido Pantotênico 11mg; 6mg Cu, 0,10mg Co, 1mg I, 50mg Fe, 65mg Mn, 45mg Zn, 0,21mg Se; Cloreto de colina 50\% 500mg; Metionina 1,4g, Agente Anticoccidiano, 60mg, Antibiótico 60mg, Antioxidante BHT 12mg. ${ }^{2}$ - de acordo com ROSTAGNO et al. (2000).
O ensaio teve duração de 10 dias, sendo cinco dias para a adaptação às dietas experimentais e cinco para a coleta das excretas. Após o período de adaptação, foi iniciada a coleta das excretas, utilizando óxido férrico (1\%) na ração, como marcador do início e do final da coleta. Durante todo o período experimental, o intervalo entre coletas foi de 12 horas. As excretas foram acondicionadas em sacos plásticos, devidamente identificadas por repetição e armazenadas a $-20^{\circ} \mathrm{C}$ após cada coleta. No final do período experimental, foram determinadas, por repetição, a quantidade de ração consumida e a quantidade total de excretas produzidas.

Para a determinação da amostra seca ao ar das excretas, estas foram descongeladas, reunidas por repetição, homogeneizadas, pesadas e colocadas em estufa de ventilação forçada, à temperatura de $55^{\circ} \mathrm{C}$, por 72 horas. Após a pré-secagem, as amostras foram moídas e acondicionadas em recipientes plásticos para análises posteriores de matéria seca, energia bruta e nitrogênio, seguindo a metodologia descrita por SILVA \& QUEIROZ (2002). Após a determinação dos valores de matéria seca, energia bruta e nitrogênio das rações e excretas, foram calculados os valores de energia metabolizável aparente (EMA) e energia metabolizável aparente corrigida para balanço de nitrogênio (EMAn), utilizando as fórmulas propostas por MATTERSON et al. (1965).

Ensaio 2 - Determinação dos coeficientes de digestibilidade dos aminoácidos

Para determinação dos coeficientes de digestibilidade dos aminoácidos, foi utilizado o método da alimentação forçada, também conhecido como alimentação precisa (SIBBALD, 1976), com galos Leghorne adultos cecectomizados, com 21 meses de idade e peso médio de $2132 \pm 257 \mathrm{~g}$. Os galos foram cecectomizados por meio de laparotomia abdominal e anestesia local, conforme a metodologia descrita por PUPA et al. (1998). Segundo ALBINO (1991), um dos fatores que interferem na digestibilidade dos aminoácidos é a atividade da microflora do intestino. O autor questiona se a microflora intestinal pode modificar a composição da excreta por degradação dos aminoácidos ou síntese de compostos nitrogenados que serão excretados. Uma alternativa, segundo CRISSEY \& THOMAS (1987), seria a utilização de galos cecectomizados, uma vez que o ceco é o local de maior atividade microbiana.

O delineamento experimental foi inteiramente casualizado, com três tratamentos, sendo dois alimentos (farelo de arroz integral e quirera de arroz) e um jejum (sem alimento), seis repetições e um galo por unidade experimental, num total de 18 galos. Os galos 
foram alojados em gaiolas individuais de baterias metálicas e, durante um período de adaptação de cinco dias, receberam alimentação em dois turnos de uma hora cada, sendo um pela manhã e o outro à tarde, com o objetivo de dilatar o papo. Posteriormente, foram submetidos a um período de jejum de 24 horas, para ocorrer o esvaziamento completo do aparelho digestivo. Após esse período, os animais foram alimentados com 30 gramas dos alimentos para testagem (15g às $8 \mathrm{~h}$ e $15 \mathrm{~g}$ às $16 \mathrm{~h})$, por intermédio de um funil-sonda introduzido via oral até o papo, a fim de evitar regurgitação do material-teste. Paralelamente, foram alojados nas mesmas condições seis galos em jejum por um período de 56 horas, para realização das correções correspondentes às excreções basais e endógenas/metabólicas.

A coleta total de excretas foi feita em intervalos de 12h, em bandejas revestidas com plásticos, acondicionadas ao piso das gaiolas de cada galo por um período de 56h após o fornecimento da primeira porção do alimento. Uma vez coletadas, as excretas foram acondicionadas em sacos plásticos, identificadas por repetição e congeladas. No final do período experimental, foi determinado o total de excreta produzida. Após serem descongeladas, as amostras foram secas como descrito no experimento 1 .

Foram determinados os coeficientes de digestibilidade verdadeira de cada aminoácido, com base nos resultados das análises de aminoácidos ingeridos e excretados e do endógeno obtido, com galos em jejum, utilizando a fórmula descrita por ROSTAGNO \& FEATHERSTON (1977).

\section{RESULTADOS E DISCUSSÃO}

Os valores da composição química e de energia metabolizável aparente e energia metabolizável aparente corrigida do farelo de arroz integral (FAI) e da quirera de arroz (QA) estão apresentados na tabela 2. O nível de MS do FAI (88,6\%) foi semelhante aos valores citados por CONTE et al. (2002) e ROSTAGNO et al. (2005), que foram de respectivamente $89,98 \%$ e $89,30 \%$, e superior ao valor de $87,25 \%$, o qual foi encontrado por GENEROSO et al. (2008). A QA apresentou valor de MS superior (93,52\%) aos valores de $86,32 \%$, 88,04\% e 86,96\% relatados por LIMA et al. (2000), ROSTAGNO et al. (2005) e GENEROSO et al. (2008), respectivamente. Segundo CONALGO et al. (1979) e ALBINO et al. (1986), a variação no teor de matéria seca pode ser atribuída às condições inadequadas de armazenamento dos cereais.

Os teores de $11,79 \%$ de $\mathrm{PB}, 15,30 \%$ de EE e 10,20 de FB do FAI foram semelhantes aos encontrados
Tabela 2 - Composição química e valores de energia do farelo de arroz integral (FAI) e da quirera de arroz (QA) em \% da matéria natural.

\begin{tabular}{lll}
\hline & FAI & QA \\
\hline Matéria seca (\%) & 88,60 & 93,52 \\
Proteína bruta (\%) & 11,79 & 9,11 \\
Extrato etéreo (\%) & 15,30 & 0,73 \\
Fibra bruta (\%) & 10,20 & 0,45 \\
Matéria mineral (\%) & 9,75 & 0,87 \\
Cálcio (\%) & 0,14 & 0,03 \\
Fósforo total (\%) & 0,97 & 0,19 \\
Sódio (\%) & 0,02 & 0,03 \\
Energia bruta (kcal kg ${ }^{-1}$ ) & 4314 & 3753 \\
Energia metabolizável aparente (kcal kg $\left.{ }^{-1}\right)$ & 2968 & 3338 \\
Energia metabolizável aparente corrigida & 2804 & 3239 \\
(kcal kg $^{-1}$ ) & & \\
\hline
\end{tabular}

por LIMA et al. (2000), que foram de respectivamente $11,54 \%, 15,30 \%$ e 10,98\%. Porém, para PB, foram inferiores aos relatados por ROSTAGNO et al. (2000), CONTE (2002), ROSTAGNO et al. (2005) e GENEROSO et al. (2008), os quais encontraram valores de 13,21\%, $16,59 \%, 13,24 \%$ e 12,34\%, respectivamente. Para a FB, foram inferiores aos $11,40 \%$ mencionados no NRC (1994) e superiores aos citados por CONTE et al. (2002) e ROSTAGNO et al. (2005), que foram de 9,68\% e 7,88\%, respectivamente. Quanto ao teor de EE, foram superiores quando comparados aos teores de $13 \%$, relacionados pelo NRC (1994), e de 14,8\%, relacionados por ROSTAGNO et al. (2005). A QA apresentou 9,11\% de $\mathrm{PB}$, valor superior aos valores encontrados na literatura, ou seja, LIMA et al. (2000), ROSTAGNO et al. (2005), BRUM et al. (2005) e GENEROSO et al. (2008) citam valores de $8,42 \%, 8,47 \%, 7,31 \%$ e $8,40 \%$, respectivamente. $\mathrm{O}$ valor de $\mathrm{EE}(0,73 \%)$ foi semelhante ao encontrado por LIMA et al. (2000), mas inferior aos valores encontrados por ROSTAGNO et al. (2005), de $1,22 \%$ e por GENEROSO et al. (2008), de $1,18 \%$, fato que justifica o valor menor de EB encontrado.

O FAI apresentou valor semelhante em relação ao cálcio $(0,14 \%)$ quando comparado ao valor de $0,13 \%$, encontrado por GENEROSO et al. (2008), mas superior aos valores encontrados por LIMA et al. (2000), CONTE et al. (2002), BRUM et al. (2005) e ROSTAGNO et al. (2005), que foram respectivamente de $0,03 \%, 0,09 \%, 0,03 \%$ e 0,11. Com relação ao fósforo total (0,97\%), o valor encontrado foi inferior quando comparado aos valores de 1,87\%, 2,06\%, 1,61\% e 1,70\%, encontrados, respectivamente, por LIMA et al. (2000), BRUM et al. (2005), ROSTAGNO et al. (2005) e GENEROSO et al. (2008). Quanto ao valor de EMAn encontrado, observou-se que este foi $12,50 \%, 9,63 \%$, $17,70 \%$ e $12,77 \%$ superior aos valores relatados por 
ROSTAGNO et al. (2000), CONTE (2002), BRUM et al. (2005) e GENEROSO et al. (2008), respectivamente, e 6,27\% inferior em relação ao NRC (1994). Todavia, essas diferenças eram esperadas, pois existem variações nas condições de solo, clima e entre cultivares. Além disso, os subprodutos são obtidos em diversas condições de processamento e manejo, que podem afetar sua composição.

O valor de EMAn da quirera de arroz foi $7,7 \%$ e $14,1 \%$ maior que o encontrado no NRC (1994) e por BRUM et al. (2005), respectivamente, e semelhante ao observado por ROSTAGNO et al. (2000) e GENEROSO et al. (2008). Tais diferenças podem estar associadas à composição química dos subprodutos do arroz, já que a energia metabolizável do alimento é um produto resultante da transformação dos nutrientes, sendo afetada direta e positivamente pela composição do alimento em carboidratos de reserva, gordura, proteína e negativamente pelos carboidratos estruturais (CONTE et al., 2002). Outros fatores, como a idade do animal e a metodologia utilizada, também afetam os valores de energia metabolizável (MARCH et al., 1973).

O valor de EMA do farelo de arroz obtido (2.968 $\mathrm{kcal} \mathrm{kg}^{-1}$ ) está próximo dos valores citados pelo NRC (1994), por ALBINO (1991) e por GIACOMETTI (2002), que foram de $2.990 \mathrm{kcal} \mathrm{kg}^{-1}$, 2.860 $\mathrm{kcal} \mathrm{kg}^{-1} \mathrm{e}$ $2.897 \mathrm{kcal} \mathrm{kg}^{-1}$, respectivamente. Entretanto, são superiores aos citados pela EMBRAPA (1991), por
ROSTAGNO et al. (1994), por CONTE (2000), por ROSTAGNO et al. (2000) e por CONTE et al. (2002), que foram de $2.518 \mathrm{kcal} \mathrm{kg}^{-1}$, 2.090 $\mathrm{kcal} \mathrm{kg}^{-1}$, 2.534kcal kg-1, $2.453 \mathrm{kcal} \mathrm{kg}^{-1}$ e $2.552 \mathrm{kcal} \mathrm{kg}^{-1}$ respectivamente. Tais diferenças parecem estar associadas à composição química do farelo de arroz, já que a energia metabolizável do alimento é afetada direta e positivamente pela composição do alimento em carboidratos de reserva (amido), gordura, proteína e negativamente pelos carboidratos estruturais (fibra). Segundo TORIN (1991), o conteúdo em amido do FAI pode variar entre 10 e $20 \%$, a gordura, entre 10 e $15 \%$, e a proteína bruta, entre 10 e $15 \%$. Assim, são esperados valores de energia metabolizável diferentes, tendo em vista essa composição.

Os valores de EMA foram superiores aos de EMAn em consequência do balanço positivo de nitrogênio, caracterizado pela retenção de nitrogênio do alimento. Isso, segundo ALBINO (1991), é esperado quando as aves têm um consumo adequado de alimento, o que ocorreu no presente estudo.

Na tabela 3, encontram-se os valores dos coeficientes de digestibilidade dos aminoácidos essenciais e não essenciais. Com exceção da lisina, metionina e arginina, que apresentaram coeficientes acima de 80,0\%, os demais aminoácidos essenciais do farelo de arroz tiveram digestibilidade inferior a 79,7\%, e o valor mais elevado foi observado para a metionina.

Tabela 3 - Valores dos aminoácidos totais (AAT), dos coeficientes de digestibilidade (CD) e dos aminoácidos digestíveis (AAD) do farelo de arroz (FAI) e da quirera de arroz (QA) com base na matéria natural.

\begin{tabular}{|c|c|c|c|c|c|c|}
\hline & \multicolumn{3}{|c|}{--------------------------FAI-------------------------- } & \multicolumn{3}{|c|}{ 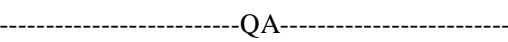 } \\
\hline & AAT & Coef. dig. & AAD & $\%$ & Coef. Dig. & AAD \\
\hline Arginina & 0,90 & 81,24 & 0,73 & 0,70 & 86,72 & 0,61 \\
\hline Fenilalanina & 0,68 & 70,77 & 0,48 & 0,36 & 64,30 & 0,23 \\
\hline Glicina & 0,66 & 79,66 & 0,53 & 0,39 & 69,54 & 0,27 \\
\hline Histidina & 0,32 & 77,23 & 0,25 & 0,23 & 83,52 & 0,19 \\
\hline Isoleucina & 0,38 & 69,08 & 0,26 & 0,33 & 77,23 & 0,25 \\
\hline Leucina & 0,86 & 71,98 & 0,62 & 0,77 & 80,00 & 0,62 \\
\hline Lisina & 0,66 & 80,10 & 0,53 & 0,39 & 80,40 & 0,31 \\
\hline Metionina & 0,21 & 86,26 & 0,18 & 0,22 & 81,94 & 0,18 \\
\hline Treonina & 0,43 & 72,51 & 0,31 & 0,33 & 77,47 & 0,26 \\
\hline Triptofano & 0,15 & 77,00 & 0,11 & 0,10 & 78,60 & 0,08 \\
\hline Valina & 0,59 & 70,29 & 0,41 & 0,48 & 77,66 & 0,37 \\
\hline Não essenciais (\%) & AAT & Coef. dig. & AAD & AAT & Coef. Dig. & AAD \\
\hline Ácido aspártico & 1,13 & 70,64 & 0,80 & 0,80 & 80,85 & 0,65 \\
\hline Ácido glutâmico & 1,60 & 76,13 & 1,22 & 1,51 & 80,01 & 1,21 \\
\hline Alanina & 0,69 & 70,39 & 0,49 & 0,41 & 74,19 & 0,30 \\
\hline Cistina & 0,25 & 77,62 & 0,19 & 0,21 & 72,65 & 0,15 \\
\hline Prolina & 0,46 & 75,89 & 0,35 & 0,50 & 76,00 & 0,38 \\
\hline Serina & 0,52 & 72,87 & 0,38 & 0,45 & 75,28 & 0,34 \\
\hline
\end{tabular}


Comparando os coeficientes de digestibilidade dos aminoácidos do farelo de arroz obtidos neste estudo com a literatura, pode-se observar uma variabilidade para os aminoácidos avaliados, exceto para o triptofano, cujo valor foi semelhante ao citado por ROSTAGNO et al. (2005).

O coeficiente de digestibilidade da arginina foi o mais alto dentre os aminoácidos da quirera de arroz, com média de $86,72 \%$, e da glicina o mais baixo, com média de 69,54\%. Os coeficientes de digestibilidade da metionina e treonina foram, respectivamente, $81,94 \%$ e $77,47 \%$, e a lisina apresentou coeficiente de digestibilidade menor ao apresentado por ROSTAGNO et al. (2005).

O FAI e a QA apresentaram, quanto à composição em aminoácidos essenciais digestíveis (Tabela 3), valores, em média, inferiores aos aminoácidos não essenciais. Esses resultados são devidos aos menores valores apresentados para os aminoácidos totais. Segundo SAUER \& OZIMEK (1986), vários fatores afetam a digestibilidade de aminoácidos dos alimentos, sendo os mais comuns s condições de processamento, a presença de compostos antinutricionais, as composições química e física da proteína e o nível de fibra. Os fatores antinutricionais nos alimentos reduzem a digestibilidade dos aminoácidos ao interferir nos processos de digestão e absorção.

É importante conhecer a digestibilidade dos aminoácidos dos diferentes ingredientes para a formulação das rações, pois a maior parte dos ingredientes apresenta digestibilidade dos aminoácidos inferior a $100 \%$, havendo uma variação considerável dependendo do tipo de ingrediente. Portanto, a concentração de aminoácidos totais não é um bom indicador do valor nutricional (PARSONS, 1991).

\section{CONCLUSÃO}

O farelo de arroz integral e a quirera de arroz podem ser utilizados em rações para frangos de corte. No entanto, deve-se atentar para seus valores inferiores em energia e superiores em proteína bruta e aminoácidos digestíveis.

\section{AGRADECIMENTOS}

À Fundação de Amparo à Pesquisa do Estado de São Paulo (Fapesp), pelo auxílio financeiro concedido (Processo 03/01989-4).

\section{REFERÊNCIAS}

ALBINO, L.F.T. et al. Energia metabolizável e composição química de alguns alimentos para frangos de corte. Revista Brasileira de Zootecnia, Viçosa, v.15, n.3, p.184-192, 1986.
ALBINO, L.F.T. Sistema de avaliação nutricional de alimentos e suas aplicações na formulação de rações para frangos de corte. 1991. 141f. Tese (Doutorado em Nutrição de Monogástricos) - Universidade Federal de Viçosa, Viçosa, MG.

ALI, M.M. et al. Investigation on rice bran: composition of rice bran and its oil. Journal of Scientific and Industrial Research, Bangladesh, v.33, n.2, p.170-177, 1998.

BRUM, P.A.R. et al. Determinação dos valores de composição química e da energia metabolizável de ingredientes para aves. Revista Brasileira de Ciência Avícola, Campinas, v.1, n.3, p.187-192, 1999.

BRUM, P.A.R. et al. Composição química e energia metabolizável de ingredientes para aves. Pesquisa Agropecuária Brasileira, Brasília, v.35, n.5, p.995-1002, 2000. Disponível em: <http://www.scielo.br/pdf/pab/v35n5/ 4721.pdf $>$. Acesso em: 15 dez. 2008. doi: 10.1590/S0100204X2000000500017.

BRUM, P.A.R. et al. Composição química e energia metabolizável de óleos, arroz vermelho e subprodutos do arroz visando a alimentação de aves. Concórdia: Embrapa Suínos e Aves, 2005.5p. (Comunicado Técnico 401). Disponível em: <www.cnpsa.embrapa.br/sgc/sgc_publicacoes/ publicacao_f0q 80p4k.pdf>. Acesso em: 12 dez. 2008.

CAFÉ, M.B.; et al. Composição e digestibilidade dos aminoácidos das sojas integrais processadas para aves. Revista Brasileira de Ciência Avícola, Campinas, v.2, n.1, p.59-66, 2000. Disponível em: <http://www.scielo.br/scielo.php?script=sci_arttext\&pid=S15 16635X2000000100009\&lng=en\&nrm=iso>. Acesso em: $15 \mathrm{dez}$. 2008. doi: 10.1590/S1516-635X2000000100009.

COLNAGO, G.L. et al. Composição química e energia metabolizável de alguns alimentos para poedeiras. Revista Brasileira de Zootecnia, Viçosa, v.80, n.4, p.654-664, 1979.

CONTE, A.J. Valor nutritivo do farelo de arroz em dietas para frangos de corte suplementadas com fitase e xilanase. 2000. 157f. Tese (Doutorado em Zootecnia) Universidade Federal de Lavras, Lavras, MG.

CONTE, A.J. et al. Efeito da fitase e xilanase sobre a energia metabolizável do farelo de arroz integral em frangos de corte. Ciência e Agrotecnologia, Lavras, v.26, n. 6, p.1289-1296, 2002. Disponível em: <http://www.editora.ufla.br/revista/26_6/ art23.PDF>. Acesso em: 10 dez. 2008.

CRISSEY, S.D.; THOMAS, O.P. Comparison of the sensitivities of growth and digestibility studies using intact, cecoctomized, and cannulated roosters. Poultry Science, Savoy, v.66, p.866874, 1987.

EMPRESA BRASILEIRA DE PESQUISA AGROPECUÁRIA. Centro Nacional de Pesquisa de Suínos e Aves. Tabela de composição química e valores energéticos de alimentos para suínos e aves. 3.ed. Concórdia, SC: Embrapa, 1991. 97p. (Documento, 19).

GENEROSO, R.A.R. et al. Composição química e energética de alguns alimentos para frangos de corte em duas idades. Revista Brasileira de Zootecnia, Viçosa, v.37, n.7, p.1251-1256, 2008. Disponível em: <http://www.scielo.br/scielo.php?script=sci_arttext\&pid=S151635982008000700016\&lng=en\&nrm=iso>. Acesso em: $12 \mathrm{dez} .2008$. doi: 10.1590/S1516-35982008000700016. 
GIACOMETTI, R.A. Valores energéticos e digestibilidade de nutrientes do farelo de arroz integral suplementado com complexos enzimáticos para frangos de corte. 2002. 54f. Dissertação (Mestrado em Zootecnia) - Universidade Federal de Lavras, Lavras, MG.

LIMA, G.J.M.M. et al. Composição química e valores de energia de subprodutos do beneficiamento de arroz. Concórdia: Embrapa Suínos e Aves, 2000. 2p. (Comunicado Técnico 244). Disponível em: <www.cnpsa.embrapa.br/sgc/ sgc_publicacoes/cot244.pdf>. Acesso em: 15 nov. 2008.

MARCH, B.E. et al. Variation in estimates of the metabolizable energy value of rapessed meal determined with chickens of different ages. Poultry Science, Savoy, v.52, p.614-618, 1973.

MATTERSON, L.S. et al. The metabolizable energy of feed ingredients for chickens. Connecticut: Agricultural Experiment Station Research Report, University of Connecticut Storrs, 1965. 11p.

NATIONAL RESEARCH COUNCIL - NRC. Nutrient requirements of poultry. 9.ed. Washington, D.C.: National Academy of Sciences, 1994. 155p.

PARSONS, C.M. Disponibilidad de aminoácidos em alimentos para aves. México: Asociación Americana de Soya, 1991. 8p. (Buletim Técnico, 101).

PARSONS, C.M. Digestible amino acids for poultry and swine. Animal Feed Science and Technology, Netherlands, v.59 n. 1-3, p.147-153, 1996.

PUPA, J.M.Ret al. Cecectomia em galos sob anestesia local e incisão abdominal. Arquivo Brasileiro de Medicina Veterinária e Zootecnia, Belo Horizonte, v.50, n.5, p.531535, 1998.

RAMALHO, R.M. Efeito da adição de enzima betaglucanase nos valores de aminoácidos digestíveis e de energia metabolizável no triticale para aves. 1998. 63f. Dissertação (Mestrado em Nutrição de Monogástricos) Universidade Federal de Viçosa, Viçosa, MG.
RODRIGUES, P.B. et al. Aminoácidos digestíveis verdadeiros da soja e subprodutos, determinados com galos cecectomizados. Revista Brasileira de Zootecnia, Viçosa, v. 31, n. 2, p. 970981, 2002. Disponível em: <http://www.revistasbz.org.br/scripts/ revista/sbz1/Artigos/3465.PDF>. Acesso em: 10 nov. 2008.

ROSTAGNO, H.S.; FEATHERSTON, W.R. Estudos de métodos para determinação de disponibilidade de aminoácidos. Revista Brasileira de Zootecnia, Viçosa, v.6, p.64-76, 1977.

ROSTAGNO, H.S. et al. Composição de alimentos e exigências nutricionais para suínos e aves. Viçosa: UFV, 1994. 59p.

ROSTAGNO, H.S. et al. Tabelas brasileiras para aves e suínos: composição de alimentos e exigências nutricionais. Viçosa, MG: UFV, 2000. 141p.

ROSTAGNO, H.S. et al. Tabelas brasileiras para aves e suínos: composição de alimentos e exigências nutricionais. 2.ed. Viçosa, MG: UFV, 2005. 186p.

SAUER, W.C.; OZIMEK, L. Digestibility of amino acids in swine: results and their practical applications. A review. Livestock Production Science, Netherlands, v.15, n.4, p.367388, 1986.

SIBBALD, I.R. A bioassay for the true metabolizable energy in feedstuffs. Poultry Science, Savoy, v.55, p.303-308, 1976.

SILVA, D.J; QUEIROZ, A.C. Análise de alimentos: métodos químicos e biológicos. 3.ed. Viçosa: MG: Universidade Federal de Viçosa, 2002. 235p.

TORIN, H.R. Utilização do farelo de arroz industrial: composição e valor nutrificante em dietas recuperativas. 1991. 147f. Dissertação (Mestrado em Ciência da Nutrição) Universidade Estadual de Campinas, Campinas, SP.

VIEITES, F.M. et al. Valores de aminoácidos digestíveis verdadeiros da farinha de carne e ossos para aves. Revista Brasileira de Zootecnia, Viçosa, v. 29, n.8, p. 2300-2307, 2000. Disponível em: <http://www.revistasbz.org.br/scripts/ revista/sbz1/Artigos/2725.PDF>. Acesso em: 15 dez. 2008. 